\title{
A note on the Moll-Arias de Reyna integral
}

\author{
M. L. Glasser ${ }^{1,2}$ (D)
}

Received: 1 May 2018 / Accepted: 12 September 2018 / Published online: 13 February 2019

(c) Springer Science+Business Media, LLC, part of Springer Nature 2019

\section{Abstract}

The Moll-Arias de Reyna integral

$$
\int_{0}^{\infty} \frac{\mathrm{d} x}{\left(x^{2}+1\right)^{3 / 2}} \frac{1}{\sqrt{\varphi(x)+\sqrt{\varphi(x)}}} \text { where } \varphi(x)=1+\frac{4}{3}\left(\frac{x}{x^{2}+1}\right)^{2}
$$

is generalized and several values are given.

Keywords Definite integral · Elliptic integral · Elliptic modulus · Algebraic integrand

Mathematics Subject Classification Primary 33E05, 33E20

\section{Introduction}

We define

$$
f(a, b)=\int_{0}^{\infty} \frac{\mathrm{d} x}{\left(x^{2}+1\right)^{a}} \frac{1}{\sqrt{\varphi(x)+\sqrt{\varphi(x)}}}
$$

where

$$
\varphi(x)=1+4 b^{-2} u^{2}, \quad u=\frac{x}{x^{2}+1} .
$$

Partial financial support is acknowledged to the Spanish Junta de Castilla y León (VA057U16) and MINECO (Project MTM2014-57129-C2-1-P).

M. L. Glasser

larryg@clarkson.edu

1 Departamento de Física Teórica, Atómica y Óptica, Facultad de Ciencias,

University of Valladolid, Valladolid 40007, Spain

2 Department of Physics, Clarkson University, Potsdam, NY 13676, USA 
The value $f(3 / 2, \sqrt{3})=\frac{\pi}{2 \sqrt{6}}$ appeared as entry 3.248.5 in [5] and was shown to be incorrect by Moll et al. [1]. Unable to find the correct evaluation, the editors decided to take this entry out of later editions of the table [6]. The exact value

$$
f(3 / 2, \sqrt{3})=\frac{\sqrt{3}-1}{2} \Pi\left(\frac{\pi}{2}, 3-\sqrt{3}, 3^{-1 / 2}\right)-6^{-1 / 2} F\left(\sin ^{-1} \sqrt{2-\sqrt{3}}, 3^{-1 / 2}\right)
$$

was recently provided in a mathematical tour de force by Arias de Reyna [2]. The aim of the present note is to provide further values of (1.1) and to suggest that the incorrect value in [5] is not merely a misprint within the scope of the parametrization (1.2).

\section{Calculation}

Factor $\sqrt{\varphi(x)}$ from the denominator of the integrand of (1.1), multiply the numerator and denominator by $\sqrt{\sqrt{\varphi(x)}-1}$, change the integration variable to $u$ (note that the range of $x$ must be divided into $[0,1] \cup[1, \infty]$ ) and set $s=2 u$ to obtain

$$
\begin{aligned}
& f(a, b) \\
& \quad=2^{-a} b \int_{0}^{1} \frac{\mathrm{d} s}{s \sqrt{1-s^{2}}}\left\{\left[1+\sqrt{1-s^{2}}\right]^{a-1}+\left[1-\sqrt{1-s^{2}}\right]^{a-1}\right\} \sqrt{1-\frac{b}{\sqrt{b^{2}+s^{2}}}}
\end{aligned}
$$

Since both quadratic surds can be rationalized by the elliptic substitution $s=\operatorname{cn}(\kappa, x)$ for a suitable modulus, $f(a, b)$ should be expressible in terms of elliptic integrals for integer and half-integer values of $a$, even in the trigonometric case $\kappa=0$. For example, it is clear that

$$
f(2, b)=\frac{1}{2} f(1, b)
$$

and with the substitution $t=b / \sqrt{b^{2}+s^{2}}$

$$
f(1, b)=k \int_{\kappa}^{1} \frac{\mathrm{d} t}{(t+1) \sqrt{(1-t)\left(t^{2}-k^{2}\right)}}, \quad k=\frac{b}{\sqrt{b^{2}+1}} .
$$

which is a complete elliptic integral of the third kind which can be manipulated into standard form [4]

$$
f(1, b)=\frac{\kappa}{\sqrt{k+1}} \Pi\left(\frac{\pi}{2}, \alpha^{2}, \kappa\right)
$$


with

$$
\alpha^{2}=\frac{\sqrt{b^{2}+1}+b}{2 \sqrt{b^{2}+1}}, \quad \kappa=\sqrt{b^{2}+1}-b
$$

For $a=3,4,5, \ldots, f(a, b)$, with $x=s^{2}$, can be seen to be a multiple of $f(1, b)$ plus an integral of the form

$$
\int_{0}^{1} \frac{P(x)}{\sqrt{1-x}} \sqrt{1-\frac{b}{\sqrt{b^{2}+x}}} \mathrm{~d} x
$$

where $P$ is a polynomial. Such an integral can be transformed into a sum of elliptic integrals by the substitutions $x \rightarrow 1-x^{2}, x \rightarrow \sqrt{b^{2}+1} \sin t$. For example,

$$
f(3, b)=\frac{1}{2} f(1, b)-\frac{b^{2}}{4 k} \int_{k}^{1} \sqrt{\frac{x(x-k)}{1-x^{2}}} \mathrm{~d} x .
$$

For $a=3 / 2(2.4)$ yields

$$
f(3 / 2, b)=\frac{b}{4} \int_{0}^{\pi / 2}[\csc (t / 2)+\sec (t / 2)] \sqrt{1-\frac{b}{\sqrt{b^{2}+\sin ^{2} t}}} \mathrm{~d} t .
$$

This can be further simplified by the substitutions $\sin t=b \sin u, \sin u=x$, $\sqrt{1+x^{2}}=1 / y$ to

$$
f(3 / 2, \sqrt{3})=\frac{3}{\sqrt{8}} \sum_{ \pm} \int_{\sqrt{3} / 2}^{1} \frac{\mathrm{d} y}{\sqrt{y(1+y)\left(4 y^{2}-3\right)\left(y \pm \sqrt{4 y^{2}-3}\right)}}
$$

which may be reduced further to

$$
f(2 / 3, \sqrt{3})=\frac{3^{1 / 4}}{2} \int_{0}^{1 / \sqrt{3}} \frac{\mathrm{d} x}{X^{3 / 2} \sqrt{4-3 X^{2}}} \frac{\sqrt{X-2 x}+\sqrt{X+2 x}}{\sqrt{X+2 / \sqrt{3}}}
$$

with $X=\sqrt{x^{2}+1}$ and which offers an alternative approach to (1.3).

\section{Discussion}

Very recently a preprint by Blaschke [3] has appeared pointing out that if the nested square root in (1.1) is replaced by the three halves power the value $\pi / 2 \sqrt{6}$ is obtained. Thus the error in [5] is indeed merely a misprint. Nevertheless, we examine the possibility of reproducing this value by a specific choice of $(a, b)$ in (1.1). To keep things 
simple, take $b=i$, so that $b \sqrt{s^{2}+b^{2}}=\left(1-s^{2}\right)^{-1 / 2}$ thus eliminating one of the surds in (1.1). Then one has

$$
\begin{aligned}
f(a, i)= & 2^{-a} \int_{0}^{1} \frac{\mathrm{d} s}{s \sqrt{1-s^{2}}}\left\{\left[1+\sqrt{1-s^{2}}\right]^{a-1}+\left[1-\sqrt{1-s^{2}}\right]^{a-1}\right\} \\
& \times \sqrt{\frac{1}{\sqrt{1-s^{2}}}-1} \\
= & 2^{1-a} \int_{0}^{1}\left[\frac{\left(1+x^{2}\right)^{a-2}}{\sqrt{1-x^{2}}}+\frac{\left(1-x^{2}\right)^{a-1 / 2}}{\sqrt{1+x^{2}}}\right] \mathrm{d} x \\
= & 2^{-a} \pi\left[{ }_{2} F_{1}(1 / 2,2-a ; 1 ;-1)+\frac{\Gamma(1-a)}{\sqrt{\pi} \Gamma(a-1 / 2)}\right] .
\end{aligned}
$$

On solving $f(a, i)=\pi / 2 \sqrt{6}$ numerically one finds the two values

$$
f(0.701935 \ldots, i)=f(11.8052 \ldots, i)=\frac{\pi}{2 \sqrt{6}} .
$$

Acknowledgements The author thanks Victor Moll for informing him of [2,3].

\section{References}

1. Amdeberhan, T., Moll, V.: The integrals in Gradshteyn and Ryzhik. Part 14: an elementary evaluation of entry 3.411.5. Scientia 19, 97-103 (2010)

2. Arias-de Reyna, J.: True value of an integral in Gradshteyn and Ryzhik's table (2018). arxiv:1801.0964v1 [math.CA]

3. Blaschke, P.: Hypergeometric form of fundamental theorem of Calculus (2018). arxiv:1808.04837v1 [math.CA]

4. Byrd, P.F., Friedman, M.D.: Handbook of Elliptic Integrals for Engineers and Scientists, 2nd edn. Springer, Berlin (1971)

5. Gradshteyn, I.S., Ryzhik, I.M.: In: Jeffrey, A., Zwillinger, D. (eds.) Table of Integrals, Series, and Products, 6th edn. Academic Press, New York (2000)

6. Gradshteyn, I.S., Ryzhik, I.M.: In: Zwillinger, D., Moll, V. (eds.) Table of Integrals, Series, and Products, 8th edn. Academic Press, New York (2015)

Publisher's Note Springer Nature remains neutral with regard to jurisdictional claims in published maps and institutional affiliations. 